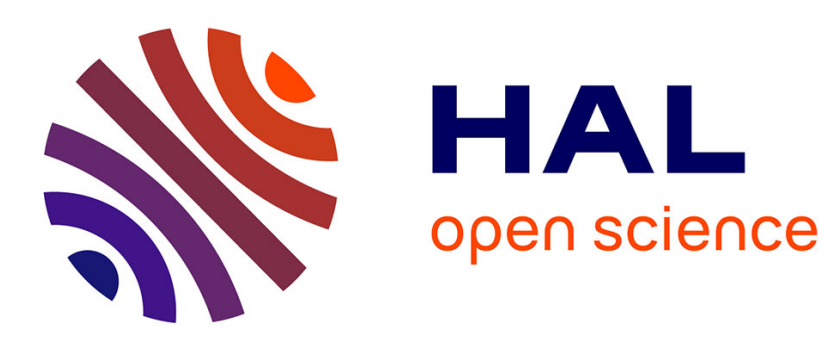

\title{
Dynamic instability of inclined cables under combined wind flow and support motion
}

\author{
Angelo Luongo, Daniele Zulli
}

\section{To cite this version:}

Angelo Luongo, Daniele Zulli. Dynamic instability of inclined cables under combined wind flow and support motion. Nonlinear Dynamics, 2012, 67 (1), pp.71-87. hal-00788394

\section{HAL Id: hal-00788394 \\ https://hal.science/hal-00788394}

Submitted on 14 Feb 2013

HAL is a multi-disciplinary open access archive for the deposit and dissemination of scientific research documents, whether they are published or not. The documents may come from teaching and research institutions in France or abroad, or from public or private research centers.
L'archive ouverte pluridisciplinaire HAL, est destinée au dépôt et à la diffusion de documents scientifiques de niveau recherche, publiés ou non, émanant des établissements d'enseignement et de recherche français ou étrangers, des laboratoires publics ou privés. 


\title{
Dynamic instability of inclined cables under combined wind flow and support motion
}

\author{
Angelo Luongo · Daniele Zulli
}

\begin{abstract}
In this paper an inclined nearly taut stay, belonging to a cable-stayed bridge, is considered. It is subject to a prescribed motion at one end, caused by traveling vehicles, and embedded in a wind flow blowing simultaneously with rain. The cable is modeled as a non-planar, nonlinear, one-dimensional continuum, possessing torsional and flexural stiffness. The lower end of the cable is assumed to undergo a vertical sinusoidal motion of given amplitude and frequency. The wind flow is assumed uniform in space and constant in time, acting on the cable along which flows a rain rivulet. The imposed motion is responsible for both external and parametric excitations, while the wind flow produces aeroelastic instability. The relevant equations of motion are discretized via the Galerkin method, by taking one in-plane and one out-of-plane symmetric modes as trial functions. The two resulting second-order, non-homogeneous, timeperiodic, ordinary differential equations are coupled and contain quadratic and cubic nonlinearities, both in the displacements and velocities. They are tackled by the Multiple Scale perturbation method, which leads to first-order amplitude-phase modulation equations, governing the slow dynamics of the cable. The wind speed, the amplitude of the support motion and the internal and external frequency detunings are set as con-
\end{abstract}

\footnotetext{
A. Luongo (凶) D. Zulli

DISAT, University of L'Aquila, Loc. Monticchio, 67100 L'Aquila (AQ), Italy

e-mail: angelo.luongo@univaq.it
}

trol parameters. Numerical path-following techniques provide bifurcation diagrams as functions of the control parameters, able to highlight the interactions between in-plane and out-of-plane motions, as well as the effects of the simultaneous presence of the three sources of excitation.

Keywords Inclined cable - Support motion . Aeroelastic instability - External excitation . Parametric excitation

\section{Introduction}

Cables are flexible and light structures, widely used in several engineering applications. Due to their natural aptitude to perform large displacements, in recent decades they have been the focus of the many researches conducted on nonlinear dynamics.

Statics and linear free dynamics of suspended horizontal and inclined cables were studied in [1-3]. Afterward, pioneering papers addressed nonlinear free dynamics, with special interest on frequency-amplitude relations and internal resonances $[4,5]$.

Nonlinear forced dynamics of cables subjected to support motions, in different resonance conditions, were studied from the theoretical [6-9] and the experimental $[10,11]$ point of view. In those papers, attention was devoted to both regular and chaotic behavior, and interactions between external and parametric excitations, induced by the support motion, were analyzed. 
On the other hand, the linear and nonlinear aeroelastic response of fixed-support horizontal cables with iced cross section, subjected to stationary wind flow causing self-excitation, was studied in [12, 13]. An attempt to roughly take into account the twist in the aerodynamic model of cables was performed in [14]. In [15-17], while considering a more realistic extension-torsion coupled constitutive law, a strongly simplified kinematic model was developed. There twist, but not bending, was accounted for, and the influence of the initial curvature on the torsion strain was ignored. Recently, the free dynamics and the modal properties of cables with bending stiffness have been analyzed in [18-20], while the bending effect on the damping of cables equipped with TMD has been studied in [21]. Complete nonlinear models were proposed in $[22,23]$; these, however, lead to very complicated equations and suffer of some numerical problems related to the existence of boundary layers, due to the smallness of the flexural terms (nearly singular equations).

In [24-26] a consistent model of cable-beam accounting for the (small) curvature of the cable, as well as for bending and torsional stiffness was formulated. By retaining only the leading terms in each equations, nonlinear reduced equations were obtained, which are identical to those of the perfectly flexible model, plus an additional equation, of static nature, accounting for both bending and torsion. The model permitted to detect the influence of the dynamic twist on the critical wind velocity. In all these papers, the aerodynamic model was taken from [27].

Dealing specifically with inclined cables, the formulation of nonlinear models, the analysis of free vibrations and the study of forced dynamics under motion of the support were reported in [28-33]. On the other hand, wind-exposed fixed-support inclined cables were studied in a stochastic [34] and a deterministic [35-37] context. In particular, wind-tunnel tests were reported in [36] for inclined cables with attached fixed or moving rain-induced rivulets, which possibly lead to galloping, induced by the asymmetry in the cross section. Furthermore, a two degree of freedom linear model was used in [37] to analyze the experimentally observed galloping of inclined cables in dry conditions.

Studies on the interaction among external, parametric and self-excitation have been carried out both in machine and structural dynamics. Among the for- mer, 1-d.o.f. systems were studied in [38-40] and $n$ d.o.f. systems were reported in [41, 42]. Among the latter, a reduced 1-d.o.f. nonlinear model of a tall building, subjected to turbulent winds, was analyzed in [43]. There, the steady part of the wind is cause of self-excitation, while the turbulent part provides both parametric and external loads.

The aim of this paper is to study the interaction among the three excitation sources (external, parametric and self-excitation) on a reduced 2-d.o.f. nonlinear model, describing the dynamics of an inclined cable belonging to a cable-stayed bridge. The model of the cable, able to twist, is taken from [26] and a sinusoidal vertical motion of given amplitude and frequency is imposed on the lower end, simulating the traffic on the deck. This is responsible for both external and parametric excitations. A uniform wind flow, blowing under a yaw angle with respect to the plane of the static configuration of the cable, is also applied. The presence of a fixed rivulet, breaking the crosssection symmetry, is accounted for by extending the aerodynamic model by Wang and $\mathrm{Xu}$ [36] to include twist. The wind is responsible for the self-excitation, as well as for modification of the parametric and external terms. The resulting equations are discretized via the Galerkin method, by assuming one in-plane and one out-of-plane modes as trial functions, accounting for the internal resonance. The two resulting secondorder, non-homogeneous, time-periodic, ordinary differential equations are coupled, containing quadratic and cubic nonlinearities, both in the displacements and velocities. They are tackled by the Multiple Scale perturbation method [44, 45], with the help of advices included in [46, 47]. Internal resonance between the two selected modes, and external resonance between the forcing and natural frequencies are accounted for. The algorithm leads to first-order amplitude-phase modulation equations, governing the slow dynamics of the cable. The wind speed, the amplitude of the support motion and the internal and external frequency detunings are set as control parameters. Numerical pathfollowing techniques provide bifurcation diagrams as functions of the control parameters, able to highlight the interactions between the three sources of excitation.

The paper is organized as follows: The equations of motion are formulated in Sect. 2. The discretization is performed in Sect. 3. The perturbation analysis is carried out in Sect. 4, where the amplitude modulation equations are derived. These latter are numerically 
sis $\left\{\overline{\mathbf{a}}_{1}, \overline{\mathbf{a}}_{2}, \overline{\mathbf{a}}_{3}\right\}$ (u is defined such that $\mathbf{x}=\overline{\mathbf{x}}+\mathbf{u}$, see Fig. 1b), and $\vartheta$ is the twist of the cross section. Displacements and twist depend on the curvilinear abscissa $s$ and on time $t$. The equations of motion were derived via the extended Hamilton principle. The boundary value problem so far obtained is not reported here, since the equations are too complicated. They, however, assume a much simpler form if an order-of-magnitude analysis is performed (see $[24,26])$ and only the leading terms are retained in each equation. The analysis is based on the following assumptions: the initial curvature $\bar{\kappa}(s)$, assumed uniform, allows one to introduce a nondimensional small parameter $\delta:=\bar{\kappa} \ell$; calling $E A, G J, E I$ the axial, torsional and bending stiffnesses respectively, the nondimensional prestress $\tau:=\bar{T} / E A$ is small of order $\delta^{3}$, where $\bar{T}$ is uniform; the nondimensional characteristic inertia radius $R=r / \ell$ of the section is also small of order $\delta^{3}$; the nondimensional stiffness parameter $\chi:=G J / E I$ is of order 1 ; the transversal displacements $v$ and $w$ are of the same order, while $\mathscr{O}(u / v)=\delta$ and $\mathscr{O}(\vartheta \ell \delta / w)=1$; the translations vary on a typical scale of length $\ell$ (since they vanish at the ends, if these are hinged) while the twist varies on a much greater length (since it does not vanish at the ends).

Consequently the nonlinear equations of motion, up to the third-order terms, become

$$
\begin{aligned}
& E A \varepsilon^{\prime}-m \ddot{u}-c_{u} \dot{u}+b_{a_{1}}=0 \\
& E A\left(\varepsilon v^{\prime}\right)^{\prime}+E A \bar{\kappa} \varepsilon+\bar{T} v^{\prime \prime}-m \ddot{v}-c_{v} \dot{v}+b_{a_{2}}=0 \\
& E A\left(\varepsilon w^{\prime}\right)^{\prime}+\bar{T} w^{\prime \prime}-m \ddot{w}-c_{w} \dot{w}+b_{a_{3}}=0 \\
& G J k_{1}^{\prime}+E I \bar{\kappa} k_{2}-\mathscr{J} \ddot{\vartheta}-c_{\vartheta} \dot{\vartheta}+c_{a_{1}}=0
\end{aligned}
$$

where the relevant strain components assume the following reduced expressions:

$$
\begin{aligned}
& \varepsilon=u^{\prime}-\bar{\kappa} v+\frac{1}{2}\left(v^{\prime 2}+w^{\prime 2}\right) \\
& k_{1}=\vartheta^{\prime}+\bar{\kappa} w^{\prime}+w^{\prime} v^{\prime \prime} \\
& k_{2}=-w^{\prime \prime}+\bar{\kappa} \vartheta+\vartheta v^{\prime \prime}
\end{aligned}
$$

In (4), $b_{a_{1}}, b_{a_{2}}, b_{a_{3}}$ and $c_{a_{1}}$ are the external forces and couple densities, $\mathscr{J}$ is the inertia polar moment of the section and $c_{u}, c_{v}, c_{w}, c_{\vartheta}$ are the viscous damping coefficients. The boundary conditions, in case of spherical hinges and vertical (direction $\mathbf{e}_{2}$ ) motion of the right support of amplitude $\eta f(t)$, read

$$
\begin{array}{lc}
u(0)=0, & u(\ell)=\eta f(t) \sin \alpha \\
v(0)=0, & v(\ell)=\eta f(t) \cos \alpha \\
w(0)=0, & w(\ell)=0 \\
G J k_{1}(0)=0, & G J k_{1}(\ell)=0
\end{array}
$$

Since the transversal-to-longitudinal (or -torsional) squared frequency ratio is small, stretching and torsion are quasi-steady, so that inertial and damping forces can be neglected in the relevant equations. Longitudinal displacements are consequently statically condensed as

$$
\begin{aligned}
u(s, t)= & \frac{\eta f(t)}{\ell} \sin \alpha-\frac{s}{\ell} \int_{0}^{\ell}\left(\bar{\kappa} v-\frac{1}{2} v^{\prime 2}-\frac{1}{2} w^{\prime 2}\right) d s \\
& +\int_{0}^{s}\left[\bar{\kappa} v(\xi, t)-\frac{1}{2} v^{\prime}(\xi, t)^{2}\right. \\
& \left.-\frac{1}{2} w^{\prime}(\xi, t)^{2}\right] d \xi
\end{aligned}
$$

where the boundary conditions (6) 1,5 have been used. In contrast, twist cannot be condensed in a such simple way, so that it is found convenient to append the relevant equation to the remaining ones. Consequently, (4) reduce to

$$
\begin{aligned}
& \frac{E A}{\ell}\left(\bar{\kappa}+v^{\prime \prime}\right)[\eta f(t) \sin \alpha \\
& \left.-\int_{0}^{\ell}\left[\bar{\kappa} v-\frac{1}{2} v^{\prime 2}-\frac{1}{2} w^{\prime 2}\right] d s\right] \\
& +\bar{T} v^{\prime \prime}+b_{2}-m \ddot{v}-c_{v} \dot{v}=0
\end{aligned}
$$

$$
\begin{aligned}
& \frac{E A}{\ell} w^{\prime \prime}\left[\eta f(t) \sin \alpha-\int_{0}^{\ell}\left[\bar{\kappa} v-\frac{1}{2} v^{2}-\frac{1}{2} w^{\prime 2}\right] d s\right] \\
& +\bar{T} w^{\prime \prime}+b_{3}-m \ddot{w}-c_{w} \dot{w}=0 \\
& G J \vartheta^{\prime \prime}-E I \bar{\kappa}^{2} \vartheta+(E I+G J) \bar{\kappa} w^{\prime \prime}-E I \bar{\kappa} \vartheta v^{\prime \prime} \\
& +G J\left(v^{\prime \prime} w^{\prime}\right)^{\prime}=0
\end{aligned}
$$

with boundary conditions $(6)_{2,4}$ and (6) 6,8 . 
Equations (8) show that, at the leading order, the dynamics of the cable are governed by the classical equations of the perfectly flexible model $(8)_{1},(8)_{2}$, usually adopted in the literature) plus an additional (quasi-static) equation $(8)_{3}$, governing the twist around the tangent. Accordingly, the twist is a slave variable, following the dynamics of the master transversal displacements. However, since the aerodynamic forces depend on the twist, this, in turn, affects the system dynamics. It is interesting to note that, while the bending does not affect the translational dynamics, it, in contrast, contributes to the twist dynamics, differently from certain models used in the literature [15-17], where it is inconsistently neglected.

\subsection{The aerodynamic model}

A simple aerodynamic model, allowing one to obtain the expressions for $b_{2}$ and $b_{3}$ in (8), is obtained as an extension of the one proposed by Wang and $\mathrm{Xu}$ [36], by adding the contribution of dynamic twist $\vartheta$. It describes the forces exerted on a rigid circular cylinder with fixed rivulet (diameter ratio $d / D=0.1$ ), inclined of an angle $\alpha$. The cylinder is capable of inplane and out-of-plane motions and is subjected to an incident uniform wind of intensity $\mathbf{U}$ and yaw angle $\beta$ (see Fig. 2a). It is based on the following simplifying assumptions: (a) the quasi-steady theory is adopted; (b) the curvature of the cable is neglected; (c) loads are evaluated taking into account the twist angle, but neglecting the (smaller) bending rotations; (d) a fixed rivulet is present on the cross section at a known phase angle; (e) the aerodynamic couples are neglected. Therefore, polynomial expressions for $b_{2}$ and $b_{3}$ in powers of $v, w, \vartheta$ are obtained, where the coefficients generally depend on the mean wind velocity.

Figure $2 b$ shows the attitudes of the cross section in the initial configuration, identified by the axes $\overline{\mathbf{a}}_{2}, \overline{\mathbf{a}}_{3}$, and in the current configuration, identified by the axes $\mathbf{a}_{2}, \mathbf{a}_{3}$, rotated by the small but finite dynamic twist $\vartheta$. The angle $\Theta_{0}$ describes the phase of the fixed rivulet. The projection of the wind velocity $\mathbf{U}$ on the plane of the cross section is denoted by $\tilde{\mathbf{U}}$ in Fig. $2 b$ and its modulus is

$$
\tilde{U}=U \sqrt{\cos ^{2}(\alpha)+\sin ^{2}(\alpha) \sin ^{2}(\beta)}
$$

where $U=\|\mathbf{U}\|$, and the angle of attack (i.e. the angle between $\tilde{\mathbf{U}}$ and a reference material axis, here taken as $\overline{\mathbf{a}}_{3}$ ) is easily obtained through geometric considerations:

$$
\begin{aligned}
\gamma: & =-\arcsin \left(\frac{\mathbf{U}}{U} \cdot \overline{\mathbf{a}}_{2}\right) \\
& =-\arcsin \left(\frac{\sin \alpha \sin \beta}{\sqrt{\cos ^{2}(\alpha)+\sin ^{2}(\alpha) \sin ^{2}(\beta)}}\right)
\end{aligned}
$$

According to the quasi-steady theory, the flow exerts on the section the aerodynamic force:

$\mathbf{b}_{a}=\frac{1}{2} \rho_{a} V r\left(c_{d}(\varphi) \mathbf{V}+c_{l}(\varphi) \mathbf{a}_{1} \times \mathbf{V}\right)$

where $\rho_{a}$ is the air density, $\mathbf{V}=\tilde{\mathbf{U}}-\dot{\mathbf{u}}=(-\tilde{U} \sin \gamma-$ $\dot{v}) \overline{\mathbf{a}}_{2}+(\tilde{U} \cos \gamma-\dot{w}) \overline{\mathbf{a}}_{3}$ is the relative velocity of the wind with respect to the center $\mathbf{G}$ of the section, $V=\|\mathbf{V}\|$ its modulus, $r$ is the radius of the cross section, $c_{d}$ and $c_{l}$ the drag and lift coefficients, respec-
Fig. 2 Inclined cylinder subjected to wind: (a) slope $\alpha$ and yaw angle $\beta$;

(b) section of the cable with fixed rivulet
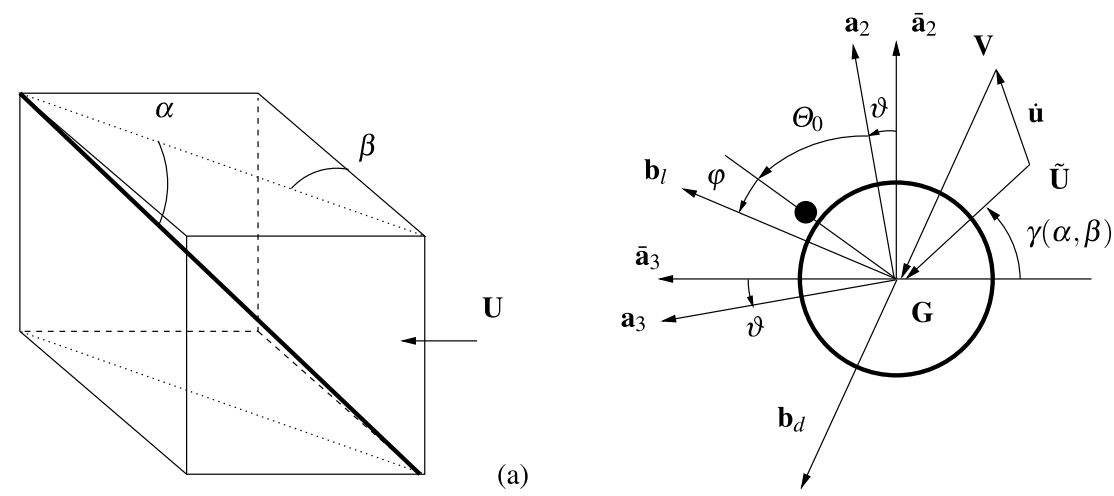
tively, depending on the angle

$$
\begin{aligned}
\varphi= & \arcsin \left(\frac{\tilde{U} \sin \gamma+\dot{v}}{\sqrt{(-\tilde{U} \sin \gamma-\dot{v})^{2}+(\tilde{U} \cos \gamma-\dot{w})^{2}}}\right) \\
& -\Theta_{0}-\vartheta
\end{aligned}
$$

defined as the difference in phase between the normal to $\mathbf{V}$ and the rivulet (see Fig. 2b). The aerodynamic coefficients, expanded up to the third power of $\varphi$, read

$$
\begin{aligned}
& c_{l}(\varphi)=\mathscr{A}_{0}+\mathscr{A}_{1} \varphi+\frac{\mathscr{A}_{2}}{2} \varphi^{2}+\frac{\mathscr{A}_{3}}{6} \varphi^{3} \\
& c_{d}(\varphi)=\mathscr{B}_{0}+\mathscr{B}_{1} \varphi+\frac{\mathscr{B}_{2}}{2} \varphi^{2}+\frac{\mathscr{B}_{3}}{6} \varphi^{3}
\end{aligned}
$$

where the coefficients $\mathscr{A}_{k}$ and $\mathscr{B}_{k}, k=1,3$, must be experimentally evaluated. It should be noticed that, following [26], the relative velocity $\mathbf{V}$ is evaluated ignoring the effect of the twist velocity $\dot{\vartheta}$, being its contribution negligible. In contrast, the twist $\vartheta$ does affect the aerodynamic forces via the angle $\varphi$ (12); consequently, $\mathbf{b}_{a}=\mathbf{b}_{a}\left(\vartheta, \dot{v}, \dot{w} ; \Theta_{0}, U\right)$. The two components of $\mathbf{b}_{a}$, along-wind $\mathbf{b}_{d}$ and cross-wind $\mathbf{b}_{l}$ are drag and lift forces, respectively (Fig. 2b).

By substituting the expressions for $\mathbf{V}$ and $\varphi$ in (11), expanding for small $\vartheta, \dot{v}$ and $\dot{w}$ up to cubic terms, and projecting on the $\overline{\mathbf{a}}_{2}, \overline{\mathbf{a}}_{3}$-axes, the following force components are derived:

$$
\begin{gathered}
b_{a_{i}}=\sum_{j=1}^{3} c_{i j}(\varphi) \xi_{j}+\sum_{j, k=1}^{3} c_{i j k}(\varphi) \xi_{j} \xi_{k} \\
+\sum_{j, k, l=1}^{3} c_{i j k l}(\varphi) \xi_{j} \xi_{k} \xi_{l}
\end{gathered}
$$

for $i=2,3$, where $\xi:=(\vartheta, \dot{v}, \dot{w})^{T}$ is the vector collecting the independent variables, $c_{i j}, c_{i j k}$ and $c_{i j k l}$ are coefficients depending on $c_{d}, c_{l}$ and their derivatives with respect to $\varphi$, all evaluated at $\overline{\mathscr{C}}$. The expressions of the coefficients, which are quite involved, are not reported here.

\subsection{Quasi-static solution}

System (8) has non-homogeneous boundary conditions. A transformation is introduced in order to obtain an equivalent system with homogeneous boundary conditions. In particular it is assumed $v(s, t):=$ $\hat{v}(s) \eta f(t)+\tilde{v}(s, t)$, where $\hat{v}(s)$ is the quasi-static component and $\tilde{v}(s, t)$ is the dynamic component of the solution. The quasi-static component is obtained as the solution of the following linearized non-homogeneous system, describing the planar linear statics of the cable when a vertical unitary time-independent motion of the support is applied:

$$
\begin{gathered}
\frac{E A}{\ell} \bar{\kappa} \sin \alpha-\frac{E A}{\ell} \bar{\kappa}^{2} \int_{0}^{\ell} \hat{v} d s+\bar{T} \hat{v}^{\prime \prime}=0 \\
\hat{v}(0)=0, \hat{v}(\ell)=\cos \alpha
\end{gathered}
$$

In particular, from (15) one obtains $\hat{v}(s)=$ $C_{1} s^{2}+C_{2} s$, where the expressions for $C_{1}$ and $C_{2}$ are

$$
\begin{aligned}
& C_{1}=\frac{3 E A m g \cos ^{3}(\alpha)\left(m g \ell \cos ^{3}(\alpha)-2 \bar{H} \sin \alpha\right)}{E A m^{2} g^{2} \ell^{3} \cos ^{5}(\alpha)+12 \bar{H}^{3} \ell} \\
& C_{2}=\frac{12 \bar{H}^{3}-2 E A m g \ell \cos ^{3}(\alpha)\left(m g \ell \cos ^{3}(\alpha)-3 \bar{H} \sin \alpha\right)}{E A m^{2} g^{2} \ell^{3} \cos ^{5}(\alpha)+12 \bar{H}^{3} \ell}
\end{aligned}
$$

A further transformation is performed to deal with nondimensional quantities. It is performed by letting

$$
\begin{aligned}
& s^{*}=\frac{s}{\ell}, \quad \omega=\frac{\pi}{\ell} \sqrt{\frac{\bar{T}}{m}}, \quad t^{*}=\omega t \\
& u^{*}=\frac{u}{\ell}, \quad v^{*}=\frac{v}{\ell}, \quad w^{*}=\frac{w}{\ell}, \quad \vartheta^{*}=\vartheta \\
& c_{n}^{*}=\frac{\omega \ell^{2}}{E A} c_{n}, \quad c_{\vartheta}^{*}=\frac{\omega}{E A} c_{\vartheta}, \\
& m^{*}=\frac{\omega^{2} \ell^{2}}{E A} m \\
& \mathscr{J}_{1}^{*}=\frac{\omega^{2}}{E A} \mathscr{J}_{1}, \quad b_{a_{i}}^{*}=\frac{\ell}{E A} b_{a_{i}}, \\
& \tau=\frac{\bar{T}}{E A}, \quad \chi=\frac{G J}{E I}, \quad c_{a_{1}}^{*}=\frac{c_{a_{1}}}{E A}
\end{aligned}
$$

where the index $n$ assumes the values $u, v, w$, and by introducing the following nondimensional control parameters:

$$
\eta^{*}=\frac{\eta}{\ell}, \quad U^{*}=\frac{U}{U_{c}}
$$


where $U_{c}$ is the critical value of the wind velocity.

Under those transformations, (8) and relevant boundary conditions become:

$$
\begin{aligned}
& \left(\hat{v}^{\prime \prime} \eta f+v^{\prime \prime}\right)[\eta f \sin \alpha \\
& \left.-\int_{0}^{1}\left[\bar{\kappa}(\hat{v} \eta f+v)-\frac{1}{2}\left(\hat{v}^{\prime} \eta f+v^{\prime}\right)^{2}-\frac{1}{2} w^{\prime 2}\right] d s\right] \\
& +\bar{\kappa} \int_{0}^{1}\left[\frac{1}{2}\left(\hat{v}^{\prime} \eta f+v^{\prime}\right)^{2}+\frac{1}{2} w^{\prime 2}\right] d s \\
& +\tau v^{\prime \prime}+b_{a_{2}}-m(\hat{v} \eta \ddot{f}+\ddot{v})-c_{v}(\hat{v} \eta \dot{f}+\dot{v})=0 \\
& w^{\prime \prime}\left[\eta f(t) \sin \alpha-\int_{0}^{1}\left[\bar{\kappa}(\hat{v} \eta f+v)-\frac{1}{2}\left(\hat{v}^{\prime} \eta f+v^{\prime}\right)^{2}\right.\right. \\
& \left.\left.\quad-\frac{1}{2} w^{\prime 2}\right] d s\right]+\tau w^{\prime \prime}+b_{a_{3}}-m \ddot{w}-c_{w} \dot{w}=0 \\
& \chi \vartheta^{\prime \prime}-\bar{\kappa}^{2} \vartheta+\left(1+\chi^{2}\right) \bar{\kappa} w^{\prime \prime} \\
& \quad-\bar{\kappa} \vartheta\left(\hat{v}^{\prime \prime} \eta f(t)+v^{\prime \prime}\right)+\chi\left(\left(\hat{v}^{\prime \prime} \eta f+v^{\prime \prime}\right) w^{\prime}\right)^{\prime}=0
\end{aligned}
$$

and:

$$
\begin{aligned}
& v(0)=0, w(0)=0, \\
& \chi\left[\vartheta^{\prime}+\bar{\kappa} w^{\prime}+w^{\prime}\left(\hat{v}^{\prime \prime} \eta f+v^{\prime \prime}\right)\right]_{s=0}=0 \\
& v(\ell)=0, w(1)=0, \\
& \chi\left[\vartheta^{\prime}+\bar{\kappa} w^{\prime}+w^{\prime}\left(\hat{v}^{\prime \prime} \eta f+v^{\prime \prime}\right)\right]_{s=1}=0
\end{aligned}
$$

where the tilde on the dynamic component and the star on the nondimensional terms have been omitted.

\section{Discretization}

A discrete model is drawn by (19)-(20) via a Galerkin procedure. The displacement field is discretized as follows: $v(s, t)=\sum_{j=1}^{M} \phi_{v_{j}}(s) q_{j}^{i}(t)$ where $q_{j}^{i}(t)$, $j=1, \ldots, M$, are the unknown amplitudes of the in-plane trial functions $\phi_{v_{j}}(s) ; w(s, t)=$ $\sum_{k=1}^{N} \phi_{w_{k}}(s) q_{k}^{o}(t)$ and $\vartheta(s, t)=\sum_{k=1}^{N} \phi_{\vartheta_{k}}(s) q_{k}^{o}(t)$ where $q_{k}^{o}(t), k=1, \ldots, N$, are the unknown amplitudes of the out-of-plane trial functions $\phi_{w_{k}}(s), \phi_{\vartheta_{k}}(s)$. The trial functions are the modes deduced from the associated linearized Hamiltonian problem. In this paper a sole in-plane, $\phi_{v}(s)$, and a sole out-of-plane, $\left\{\phi_{w}(s), \phi_{\vartheta}(s)\right\}$, eigenfunctions are selected $(M=$ $N=1)$. Two non-autonomous ordinary differential equations are obtained:

$$
\begin{aligned}
\ddot{q}_{1} & +\Gamma_{1,1} \dot{q}_{1}+\Gamma_{1,2} q_{1}+\Gamma_{1,3} q_{2}+\Gamma_{1,4} \dot{q}_{2}+\Gamma_{1,5} q_{1}^{2} \\
& +\Gamma_{1,6} q_{2}^{2} \\
& +\Gamma_{1,7} q_{1}^{3}+\Gamma_{1,8} q_{2}^{3}+\Gamma_{1,9} q_{1} q_{2}^{2}+\Gamma_{1,10} \dot{q}_{1}^{2} \\
& +\Gamma_{1,11} \dot{q}_{1} \dot{q}_{2} \\
& +\Gamma_{1,12} \dot{q}_{2}^{2}+\Gamma_{1,13} \dot{q}_{1} q_{2}+\Gamma_{1,14} \dot{q}_{2} q_{2}+\Gamma_{1,15} \dot{q}_{1}^{3} \\
& +\Gamma_{1,16} \dot{q}_{2}^{3}+\Gamma_{1,17} \dot{q}_{1}^{2} q_{2}+\Gamma_{1,18} \dot{q}_{1} q_{2}^{2}+\Gamma_{1,19} q_{2} \dot{q}_{2}^{2} \\
& +\Gamma_{1,20} q_{2}^{2} \dot{q}_{2}+\Gamma_{1,21} q_{2} \dot{q}_{1} \dot{q}_{2}+\Gamma_{1,22} \dot{q}_{1}^{2} \dot{q}_{2}=\Gamma_{1,23} \\
\ddot{q}_{2} & +\Gamma_{2,1} \dot{q}_{1}+\Gamma_{2,2} \dot{q}_{2}+\Gamma_{2,3} q_{2}+\Gamma_{2,4} q_{2}^{2}+\Gamma_{2,5} q_{1} q_{2} \\
& +\Gamma_{2,6} q_{2}^{3}+\Gamma_{2,7} q_{1}^{2} q_{2}+\Gamma_{2,8} \dot{q}_{1}^{2}+\Gamma_{2,9} \dot{q}_{2}^{2} \\
& +\Gamma_{2,10} \dot{q}_{1} q_{2}+\Gamma_{2,11} q_{2} \dot{q}_{2}+\Gamma_{2,12} \dot{q}_{1} \dot{q}_{2} \\
& +\Gamma_{2,13} q_{2}^{2} \dot{q}_{2}+\Gamma_{2,14} \dot{q}_{1} \dot{q}_{2} q_{2} \\
& +\Gamma_{2,15} q_{2}^{2} \dot{q}_{1}+\Gamma_{2,16} q_{2} \dot{q}_{1}^{2}+\Gamma_{2,17} \dot{q}_{1}^{3}+\Gamma_{2,18} \dot{q}_{1}^{2} \dot{q}_{2} \\
& +\Gamma_{2,19} q_{2} \dot{q}_{2}^{2}+\Gamma_{2,20} \dot{q}_{1} \dot{q}_{2}^{2}+\Gamma_{2,21} \dot{q}_{2}^{3}=\Gamma_{2,22}
\end{aligned}
$$

where modal damping has been added, and where $q_{1}(t)$ and $q_{2}(t)$ describe the in-plane and out-of-plane time-laws, respectively. Partial expressions of the coefficients $\Gamma_{i, j}$ are reported in Appendix A; they depend on the wind velocity $U$ and the amplitude of the support motion $\eta$.

As a general discussion on (21), self-, parametric and external excitations are noticed, and both quadratic and cubic nonlinearities appear. The twist $\vartheta$, related to the aerodynamic forces, leads to a stiffness coupling and also contributes to some nonlinear mechanical coefficients, consistently with the circulatory nature of the forces. Moreover, it appears in mixed velocity-displacement terms. It can be seen, that the simultaneous presence of the motion of the support and wind produces terms otherwise absent when one of the two sources of excitation is missing. For example $\zeta_{61} \frac{\eta f(t)}{U} \dot{q}_{1}^{2}$ in $\Gamma_{1,10}$, and $\zeta_{32} \frac{\eta f(t)}{U} \dot{q}_{1} \dot{q}_{2}$ in $\Gamma_{1,11}$, are key-terms in the subsequent analysis. 


\section{Perturbation analysis}

The Multiple Scales perturbation Method (MSM) is used on (21) to asymptotically describe the slow dynamics of the system. Since both quadratic and cubic terms occur in (21), three orders of perturbation equations are needed.

It is assumed that in-plane and out-of-plane natural frequencies are in 1:1 ratio, $\omega_{1} \simeq \omega_{2}$, i.e. the cable is 1:1 internally resonant. The support motion is taken of sinusoidal type, $f(t)=\sin (\Omega t)$, where the forcing frequency is close to the natural ones. Therefore a 1:1 external resonance occurs with both the modes, simultaneously. In order to express the closeness of the three frequencies, two detunings $\sigma_{1}$ and $\sigma_{2}$ are taken, such that $\omega_{1}=\Omega+\varepsilon^{2} \sigma_{1}, \omega_{2}=\Omega+\varepsilon^{2} \sigma_{2}$, where $\varepsilon$ is a dimensionless perturbation parameter $(\varepsilon \ll 1)$. These definitions, according to [9], are found computationally more convenient than the "classical" definitions $\Omega=\omega_{1}+\varepsilon^{2} \sigma$ and $\omega_{2}=\omega_{1}+\varepsilon^{2} \rho$ where an external $(\sigma)$ and an internal $(\rho)$ detunings appear. Of course the following relations hold between the two sets of detunings: $\sigma=-\sigma_{1}$ and $\rho=\sigma_{2}-\sigma_{1}$. Following the MSM, the dependent variables $q_{1}, q_{2}$ are expanded in series of $\varepsilon, q_{k}=\varepsilon q_{k 1}+\varepsilon^{2} q_{k 2}+\varepsilon^{3} q_{k 3}, k=1,2$ and several time scales are introduced; the structural damping is assumed small, of order $\varepsilon^{2}$; the aerodynamic terms, assumed small too, are ordered so as to appear at the third order; $\eta$ is assumed of order $\varepsilon$, while the coefficients are ordered so that the resonant external and parametric excitation terms appear at the highest-order perturbation equations.

After performing standard steps, not reported here, Amplitude Modulation Equations (AME) up to the third order are obtained. They describe the evolution in time of the complex amplitudes $A_{1}(t)$ and $A_{2}(t)$ of the variables $q_{1}$ and $q_{2}$, respectively (namely $q_{k}=$ $A_{k} \exp \left(i \omega_{k} t\right)+\bar{A}_{k} \exp \left(-i \omega_{k} t\right)+$ higher-order terms, with the over-bar denoting the complex conjugate and $i$ the imaginary unit). The AME assume the form:

$$
\begin{aligned}
& \dot{A}_{1}=F_{1}\left(A_{1}, \bar{A}_{1}, A_{2}, \bar{A}_{2} ; \eta, U, \sigma_{1}, \sigma_{2}\right) \\
& \dot{A}_{2}=F_{2}\left(A_{1}, \bar{A}_{1}, A_{2}, \bar{A}_{2} ; \eta, U, \sigma_{1}, \sigma_{2}\right)
\end{aligned}
$$

where $\eta, U, \sigma_{1}$ and $\sigma_{2}$ are control parameters. The complex equations (22) are expressed in real form by using both polar and Cartesian representation, each having specific conveniences. In polar form, by defining the real amplitudes $a_{1}(t), a_{2}(t)$ and phases
$\Phi_{1}(t), \Phi_{2}(t)$, so that $A_{1}=a_{1} \exp \left(i \Phi_{1}\right)$ and $A_{2}=$ $a_{2} \exp \left(i \Phi_{2}\right),(22) \mathrm{read}$

$\dot{a}_{1}=p_{1}\left(a_{1}, a_{2}, \Phi_{1}, \Phi_{2} ; \eta, U, \sigma_{1}, \sigma_{2}\right)$

$\dot{a}_{2}=p_{2}\left(a_{1}, a_{2}, \Phi_{1}, \Phi_{2} ; \eta, U, \sigma_{1}, \sigma_{2}\right)$

$a_{1} \dot{\Phi}_{1}=p_{3}\left(a_{1}, a_{2}, \Phi_{1}, \Phi_{2} ; \eta, U, \sigma_{1}, \sigma_{2}\right)$

$a_{2} \dot{\Phi}_{2}=p_{4}\left(a_{1}, a_{2}, \Phi_{1}, \Phi_{2} ; \eta, U, \sigma_{1}, \sigma_{2}\right)$

In Cartesian form, by defining the components $r_{1}(t), s_{1}(t), r_{2}(t), s_{2}(t)$ so that $A_{1}=r_{1}+i s_{1}$ and $A_{2}=$ $r_{2}+i s_{2}$, they read

$$
\begin{aligned}
& \dot{r}_{1}=g_{1}\left(r_{1}, s_{1}, r_{2}, s_{2} ; \eta, U, \sigma_{1}, \sigma_{2}\right) \\
& \dot{s}_{1}=g_{2}\left(r_{1}, s_{1}, r_{2}, s_{2} ; \eta, U, \sigma_{1}, \sigma_{2}\right) \\
& \dot{r}_{2}=g_{3}\left(r_{1}, s_{1}, r_{2}, s_{2} ; \eta, U, \sigma_{1}, \sigma_{2}\right) \\
& \dot{s}_{2}=g_{4}\left(r_{1}, s_{1}, r_{2}, s_{2} ; \eta, U, \sigma_{1}, \sigma_{2}\right)
\end{aligned}
$$

\section{Numerical results}

Numerical results are reported here for a sample cable (almost identical to the one presented in [33]) of length $\ell=121.9 \mathrm{~m}$, position of the right support of components $x_{\ell}=99.6 \mathrm{~m}$ and $y_{\ell}=-70.3 \mathrm{~m}$ respectively, diameter of the cross section $D=0.119 \mathrm{~m}$, diameter of the rivulet $d=0.1 D$, inclination of the chord $\alpha=-35.2^{\circ}$, axial stiffness $E A=29.7 \times 10^{6} \mathrm{~N}$, torsional stiffness $G J=159 \mathrm{Nm}^{2}$, bending stiffness $E I=2100 \mathrm{Nm}^{2}$, mass per-unit-length $m=$ $51.8 \mathrm{Kg} / \mathrm{m}$, structural damping ratio $\xi_{s}=0.4 \%$; the initial axial tension turns out to be $\bar{H}=3142 \mathrm{KN}$. The cable is very taut and the trial functions are the first in-plane symmetric mode (nondimensional natural frequency $\left.\omega_{1}=1.004\right)$ and the first out-ofplane symmetric mode (nondimensional natural frequency $\omega_{2}=1$ ), both normalized as the maximum displacements are 1 . The drag and lift coefficients are taken from [36], where they were obtained by windtunnel tests. Their values are $\mathscr{A}_{0}=-0.48, \mathscr{A}_{1}=0.84$, $\mathscr{A}_{2}=4.69, \mathscr{A}_{3}=0.86, \mathscr{B}_{0}=1.25, \mathscr{B}_{1}=-0.65$, $\mathscr{B}_{2}=-0.08, \mathscr{B}_{3}=6.81$. The critical (dimensional) wind velocity is $U_{c}=36 \mathrm{~m} / \mathrm{s}$. Equilibrium branches and periodic solutions of (22) are numerically sought using the software Auto [48], producing periodic and quasi-periodic oscillations in the variables $q_{i}$. The resulting nondimensional amplitudes $a_{1}$ and $a_{2}$, accord- 
Fig. 5 Amplitude $a_{1}$ vs.

excitation frequency, in absence of wind:
(a) $\eta=8.2 \times 10^{-5}$;
(b) $\eta=4.1 \times 10^{-4}$.

Continuous line: stable; dashed line: unstable
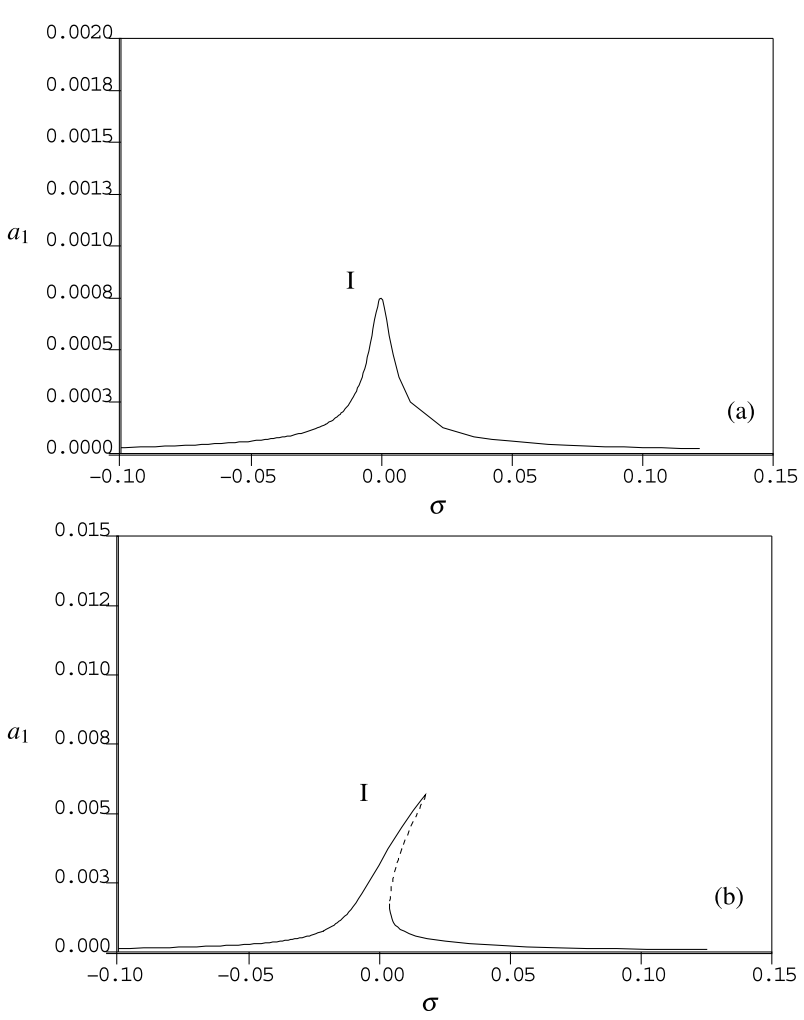

Fig. 6 Amplitudes $a_{1}$ and $a_{2}$ vs. excitation frequency, in absence of wind, when $\eta=1.4 \times 10^{-3}$. Continuous line: stable; dashed line: unstable
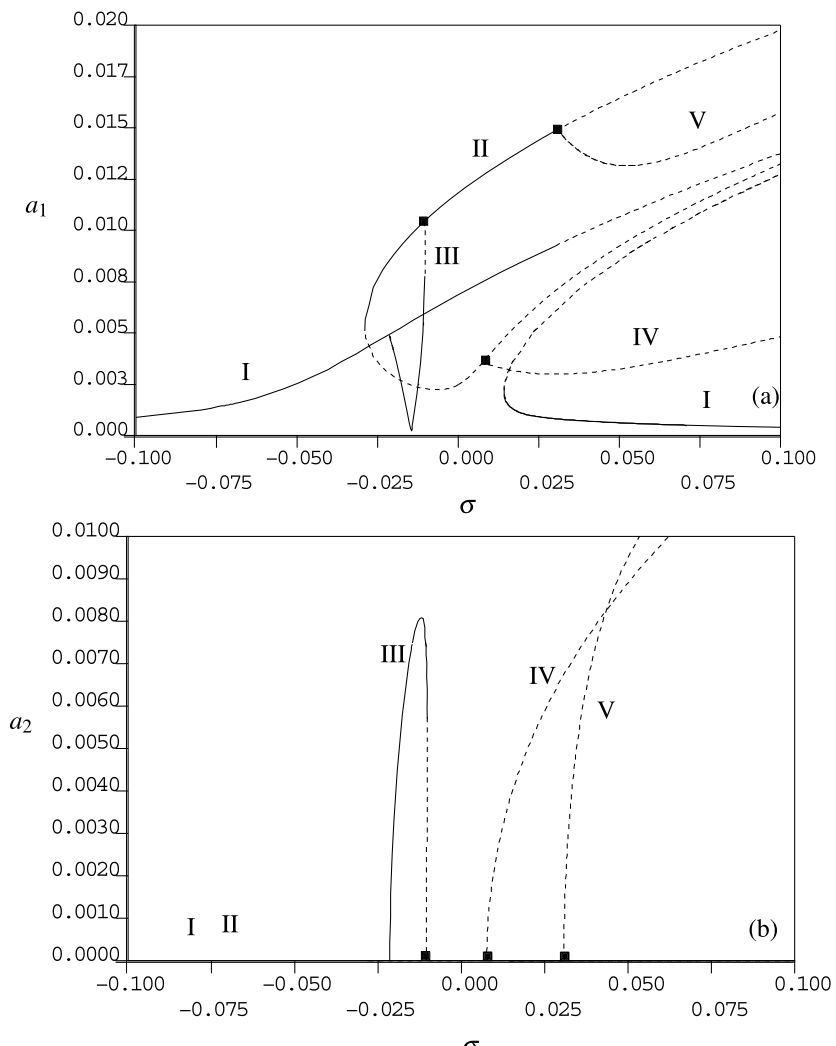
Fig. 7 Amplitudes $a_{1}$ and $a_{2}$ vs. excitation frequency, when $U=0.7$ and $\eta=4.1 \times 10^{-4}$. Continuous line: stable; dashed line: unstable

Fig. 8 Amplitudes $a_{1}$ and $a_{2}$ vs. excitation frequency, when $U=1.3$ and $\eta=4.1 \times 10^{-4}$. Continuous line: stable; dashed line: unstable
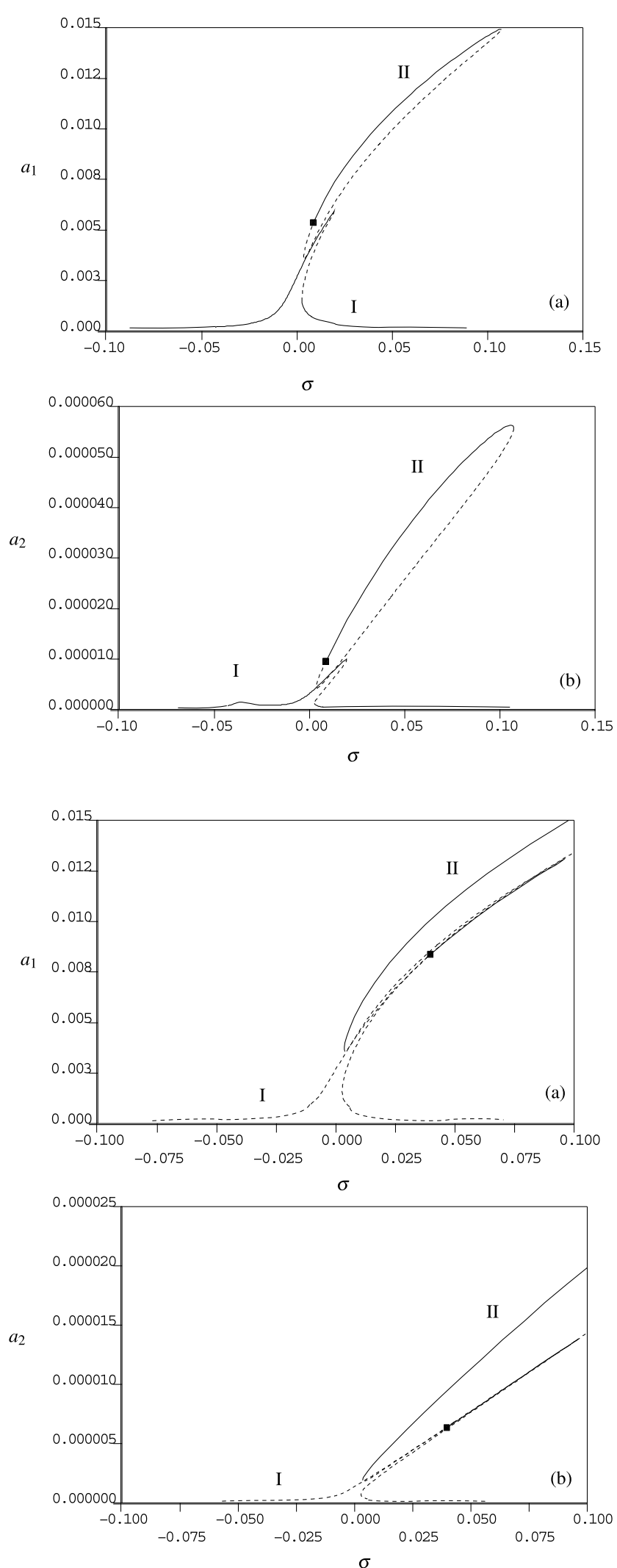
Fig. 9 Amplitudes $a_{1}$ and $a_{2}$ vs. excitation frequency, when $U=0.7$ and $\eta=1.4 \times 10^{-3}$. Continuous line: stable; dashed line: unstable; empty boxes: numerical integrations

Fig. 10 Amplitudes $a_{1}$ and $a_{2}$ vs. excitation frequency, when $U=1.3$ and $\eta=1.4 \times 10^{-3}$. Continuous line: stable; dashed line: unstable; empty boxes: numerical integrations
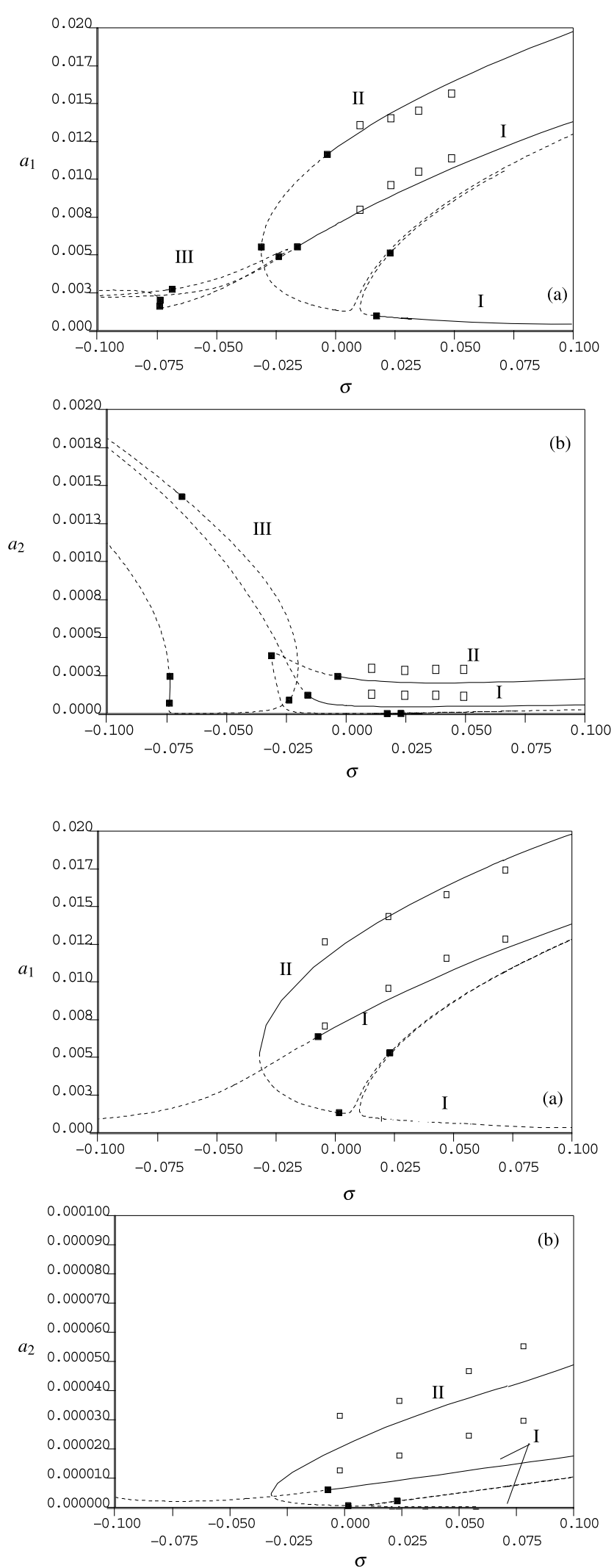
Fig. 11 Time evolution of

the amplitudes $a_{1}$ and $a_{2}$ when $\eta=1.4 \times 10^{-3}$, $\sigma=-0.042$ and $U=1.3$
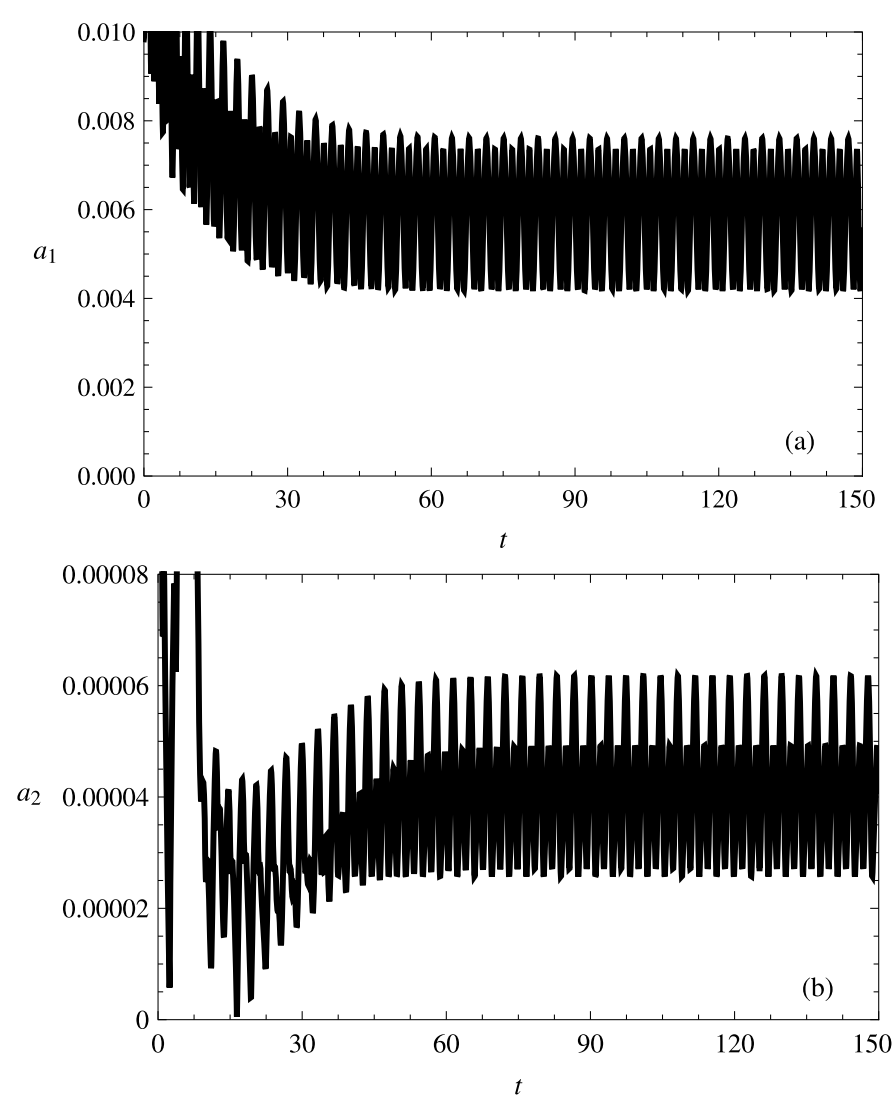

the wind increases, it drastically reduces the amplitude of the out-of-plane motion. However, wind leaves the shape of the branches I and II almost unchanged, while the extension of the stable zone and the position of the Hopf bifurcation points are modified. From these points, short, unstable branches of periodic solutions start, which quickly die at a homoclinic tangency (not reported in the figures). Other periodic solutions in the amplitudes $a_{1}, a_{2}$ are found; for example, when $\eta=1.4 \times 10^{-3}, \sigma=-0.042, U=1.3$, the evolution in time of the solution is shown in Fig. 11.

The previous result, according to which aerodynamic forces reduce out-of-plane motions, otherwise triggered by support excitation, appears as the main result of the analysis. Although the phenomenon is quite surprising, it must be ascribed to the fact, already noticed, that aerodynamic and parametric excitation interact (in an a priori unpredictable way) in modifying the coefficients of the equations of motion, or even creating new terms. This behavior has been checked by direct numerical integration of the original equations (21), whose results are marked by empty boxes in Figs. 9 and 10. A good accordance is highlighted, which corroborates the analytical findings. The same results might be obtained just considering a (simpler) 1-d.o.f. system, obtained from (22) for $A_{2}=0$ and retaining only the first equation. In this case, it can be shown that the parametric excitation due to the combined wind-support motion provides terms of the same nature of those due to both super-harmonic and sub-harmonic excitations, responsible for the existence of the island (see [44]), plus a new term due to the quadratic parametric excitation.

\section{Conclusions}

A nonlinear continuous model has been formulated to describe the non-planar dynamics of an inclined cable, subjected to stationary wind and sinusoidal motion at the lower support. Torsional and flexural stiffnesses of the cable have been accounted for in the model, by using a consistent asymptotic analysis grounded on the smallness of the sag-to-span ratio. A quite simpli- 
fied aerodynamic model of cable-rivulet has been employed, by adapting and extending a model taken from literature. A 2-d.o.f. model has been deduced by a Galerkin projection, leading to time-dependent o.d.e., expanded up to the third order. The motion enforced at the ground is found to be responsible for external and parametric excitation, while the wind causes selfexcitation; moreover, it modifies the effects of the excitation at the support. The equations have been tackled with the Multiple Scales technique, in the case of 1:1 external resonance and 1:1 internal resonance. The mechanical behavior of the cable has been described for different amplitudes of support motion, wind velocity and excitation frequency. It has been shown that, when the motion of the support has small amplitude, the galloping is perturbed, and islands of non-trivial periodic motions of large amplitude exist, also for velocities smaller than the critical one. When the motion of the support is large, remarkable out-of-plane oscillations occur in no-wind case. Those, however, and quite surprisingly, are drastically reduced in the presence of wind, due to the combined effect of the two sources of excitation. Finally, the existence of quasiperiodic motions and homoclinic bifurcations has been ascertained.

Acknowledgements This work has been partially supported by the Italian Ministry of Education, University and Research (MIUR) through a PRIN co-financed program (www.disg. uniroma1.it/fendis).

\section{Appendix A: Coefficients of the discrete equation of motion}

In this section, the partial expressions of the coefficients of (21) are reported. The in-plane and out-ofplane natural nondimensional frequencies of the cable are $\omega_{1}$ and $\omega_{2} ; \xi_{s}$ is the modal damping ratio. The coefficients $\Gamma_{i j}$ are the following:

$$
\begin{aligned}
& \Gamma_{1,1}=2 \xi_{s} \omega_{1}+\zeta_{11} U+\zeta_{12} \frac{\eta^{2} \dot{f}(t)^{2}}{U}+\zeta_{13} \eta \dot{f}(t) \\
& \Gamma_{1,2}=\omega_{1}^{2}+k_{11} \eta f(t)+k_{12} \eta^{2} f(t)^{2} \\
& \Gamma_{1,3}=k_{21} U^{2}+k_{22} \dot{f}(t)^{2} \eta^{2}+k_{23} U \dot{f}(t) \eta \\
& \Gamma_{1,4}=\zeta_{21} \eta \dot{f}(t)+\zeta_{22} U+\zeta_{23} \frac{\eta^{2} \dot{f}(t)^{2}}{U} \\
& \Gamma_{1,5}=k_{31}+k_{32} \eta f(t)
\end{aligned}
$$

$$
\begin{aligned}
& \Gamma_{1,6}=k_{51}+k_{52} \eta f(t)+k_{53} U^{2}+k_{54} U \dot{f}(t) \eta \\
& \Gamma_{1,7}=k_{4}, \quad \Gamma_{1,8}=k_{6} U^{2}, \quad \Gamma_{1,9}=k_{7} \\
& \Gamma_{1,10}=\zeta_{61} \frac{\eta \dot{f}(t)}{U}+\zeta_{62}, \\
& \Gamma_{1,11}=\zeta_{31}+\zeta_{32} \frac{\eta \dot{f}(t)}{U} \\
& \Gamma_{1,12}=\zeta_{19}+\frac{\bar{\zeta}_{22}}{U}, \quad \Gamma_{1,13}=\zeta_{41} \eta \dot{f}(t)+\zeta_{42} U \\
& \Gamma_{1,14}=\zeta_{51} U+\zeta_{52} \eta \dot{f}(t), \quad \Gamma_{1,15}=\frac{\zeta_{8}}{U}, \\
& \Gamma_{1,16}=\frac{\bar{\zeta}_{21}}{U} \\
& \Gamma_{1,17}=\bar{\zeta}_{12}, \quad \Gamma_{1,18}=\bar{\zeta}_{13} U, \quad \Gamma_{1,19}=\zeta_{14} \\
& \Gamma_{1,20}=\zeta_{15}, \quad \Gamma_{1,21}=\zeta_{16}, \quad \Gamma_{1,22}=\frac{\zeta_{17}}{U} \\
& \Gamma_{1,23}=k_{01} \eta^{2} f(t)^{2}+k_{02} \eta^{3} f(t)^{3}+\zeta_{01} U \eta \dot{f}(t) \\
& +\frac{\zeta_{02}}{U} \eta^{3} \dot{f}(t)^{3}+m_{0} \eta \ddot{f}(t)
\end{aligned}
$$

and

$$
\begin{aligned}
\Gamma_{2,1}= & \xi_{1} \eta \dot{f}(t)+\xi_{12} U+\xi_{13} \frac{\eta^{2} \dot{f}(t)^{2}}{U} \\
\Gamma_{2,2}= & 2 \xi_{s} \omega_{2}+\xi_{21} U+\xi_{22} \eta \dot{f}(t)+\xi_{23} \frac{\eta^{2} \dot{f}(t)^{2}}{U} \\
\Gamma_{2,3}= & \omega_{2}^{2}+h_{11} \eta f(t)+h_{12} \eta^{2} f(t)^{2}+h_{13} U \eta \dot{f}(t) \\
& +U^{2} h_{14}+h_{15} \eta^{2} \dot{f}(t)^{2} \\
\Gamma_{2,4}= & h_{21} U^{2}+h_{22} U \eta \dot{f}(t), \\
\Gamma_{2,5}= & h_{41}+h_{42} \eta f(t) \\
\Gamma_{2,6}= & h_{31}+h_{32} U^{2}, \\
\Gamma_{2,8}= & \xi_{61}+\xi_{62} \frac{\eta \dot{f}(t)}{U}, \\
\Gamma_{2,9}= & \xi_{71}+\xi_{72} \frac{\eta \dot{f}(t)}{U} \\
\Gamma_{2,10}= & \xi_{41} U+\xi_{42} \eta \dot{f}(t), \\
\Gamma_{2,11}= & \xi_{51} \eta \dot{f}(t)+\xi_{52} U
\end{aligned}
$$




$$
\begin{aligned}
& \Gamma_{2,12}=\xi_{81}+\xi_{82} \frac{\eta \dot{f}(t)}{U}, \quad \Gamma_{2,13}=\xi_{15} U \\
& \Gamma_{2,14}=\xi_{16}, \quad \Gamma_{2,15}=U \xi_{9}, \quad \Gamma_{2,16}=\xi_{10} \\
& \Gamma_{2,17}=\frac{\xi_{11}}{U}, \quad \Gamma_{2,18}=\frac{\xi_{12}}{U}, \quad \Gamma_{2,19}=\bar{\xi}_{13} \\
& \Gamma_{2,20}=\frac{\xi_{14}}{U}, \quad \Gamma_{2,21}=\frac{\xi_{17}}{U} \\
& \Gamma_{2,22}=\xi_{01} \eta^{2} \dot{f}(t)^{2}+\xi_{02} U \eta \dot{f}(t)+\frac{\xi_{03}}{U} \eta^{3} \dot{f}(t)^{3}
\end{aligned}
$$

The coefficients $k_{i j}, h_{i j}, \zeta_{i j}, \xi_{i j}, \bar{\zeta}_{i j}, \bar{\xi}_{i j}, m_{0}$ come from integrations of the shape functions. In particular, $k_{i j}, h_{i j}$ contain elastic parameters, $\zeta_{i j}, \xi_{i j}, \bar{\zeta}_{i j}, \bar{\xi}_{i j}$ contain aerodynamic parameters, $m_{0}$ is the modal mass. Their analytical expressions are not reported here for brevity.

\section{References}

1. Irvine, H.M., Caughey, T.K.: The linear theory of free vibrations of a suspended cable. Proc. R. Soc. Lond. A 341, 299-315 (1974)

2. Irvine, H.M.: Cable Structures. MIT Press, Cambridge (1981)

3. Triantafyllou, M.S.: The dynamics of taut inclined cables. Q. J. Mech. Appl. Math. 37(3), 421-440 (1984)

4. Hagedorn, P., Schäfer, B.: On non-linear free vibrations of an elastic cable. Int. J. Non-Linear Mech. 15(4-5), 333-340 (1980)

5. Luongo, A., Rega, G., Vestroni, F.: Monofrequent oscillations of a non-linear model of a suspended cable. J. Sound Vib. 82(2), 247-259 (1982)

6. Perkins, N.C.: Modal interactions in the non-linear response of elastic cables under parametric/external excitation. Int. J. Non-Linear Mech. 27(2), 233-250 (1992)

7. Benedettini, F., Rega, G., Alaggio, R.: Non-linear oscillations of a four-degree-of-freedom model of a suspended cable under multiple internal resonance conditions. J. Sound Vib. 182(5), 775-798 (1995)

8. Rega, G., Lacarbonara, W., Nayfeh, A.H., Chin, C.M.: Multiple resonances in suspended cables: direct versus reducedorder models. Int. J. Non-Linear Mech. 34, 901-924 (1999)

9. Chen, H., Zuo, D., Zhang, Z., Xu, Q.: Bifurcations and chaotic dynamics in suspended cables under simultaneous parametric and external excitations. Nonlinear Dyn. (2010). doi:10.1007/s11071-010-9750-2

10. Rega, G., Alaggio, R., Benedettini, F.: Experimental investigation of the nonlinear response of a hanging cable. Part I. Local analysis. Nonlinear Dyn. 14(2), 89-117 (1997)

11. Rega, G.: Nonlinear vibrations of suspended cables. Part I. Modeling and analysis. Appl. Mech. Rev. 57(6), 443-478 (2004)
12. Luongo, A., Paolone, A., Piccardo, G.: Postcritical behavior of cables undergoing two simultaneous galloping modes. Meccanica 33(3), 229-242 (1998)

13. Luongo, A., Piccardo, G.: A continuous approach to the aeroelastic stability of suspended cables in 1:2 internal resonance. J. Vib. Control 14(1-2), 135-157 (2008)

14. Luongo, A., Piccardo, G.: On the influence of the torsional stiffness on non-linear galloping of suspended cables. In: Proc. 2nd ENOC, vol. 1, pp. 273-276, Prague, Czech Republic (1996)

15. Yu, P., Desai, Y.M., Popplewell, N., Shah, A.H.: Threedegree-of-freedom model for galloping. Part II. Solutions. J. Eng. Mech. 119(12), 2426-2448 (1993)

16. Mcconnel, K.G., Chang, C.N.: A study of the axialtorsional coupling effect on a sagged transmission line. Exp. Mech. 1, 324-329 (1986)

17. White, W.N., Venkatasubramanian, S., Lynch, P.M., Huang, C.D.: The equations of motion for the torsional and bending vibrations of a stranded cabl. Appl. Mech. Div., 91WA/APM-19, 1993

18. Ricciardi, G., Saitta, F.: A continuous vibration analysis model for cables with sag and bending stiffness. Eng. Struct. 30, 1459-1472 (2008)

19. Treyssède, F.: Vibration analysis of horizontal selfweighted beams and cables with bending stiffness subjected to thermal loads. J. Sound Vib. 329, 1536-1552 (2010)

20. Ceballos, M.A., Prato, C.A.: Determination of the axial force on stay cables accounting for their bending stiffness and rotational end restraints by free vibration tests. J. Sound Vib. 317, 127-141 (2008)

21. Hijmissen, J.W., van den Heuvel, N.W., van Horssen, W.T.: On the effect of the bending stiffness on the damping properties of a tensioned cable with an attached tuned-massdamper. Eng. Struct. 31, 1276-1285 (2009)

22. Lu, C.L., Perkins, N.C.: Nonlinear spatial equilibria and stability of cables under uni-axial torque and thrust. J. Appl. Mech. 61, 879-886 (1994)

23. Diana, G., Bruni, S., Cheli, F., Fossati, F., Manenti, A.: Dynamic analysis of the transmission line crossing Lago de Maracaibo. J. Wind Eng. Ind. Aerodyn. 74-76, 977-986 (1998)

24. Luongo, A., Zulli, D., Piccardo, G.: A linear curved-beam model for the analysis of galloping in suspended cables. J. Mech. Mater. Struct. 2(4), 675-694 (2007)

25. Luongo, A., Zulli, D., Piccardo, G.: Analytical and numerical approaches to nonlinear galloping of internally resonant suspended cables. J. Sound Vib. 315(3), 375-393 (2008)

26. Luongo, A., Zulli, D., Piccardo, G.: On the effect of twist angle on nonlinear galloping of suspended cables. Compos. Struct. 87, 1003-1014 (2009)

27. Novak, M.: Aeroelastic galloping of prismatic bodies. J. Eng. Mech. 95(EM1), 115-141 (1969)

28. Srinil, N., Rega, G., Chucheepsakul, S.: Two-to-one resonant multi-modal dynamics of horizontal/inclined cables. Part I. Theoretical formulation and model validation. Nonlinear Dyn. 48(3), 231-252 (2007)

29. Srinil, N., Rega, G.: Two-to-one resonant multi-modal dynamics of horizontal/inclined cables. Part II. Internal resonance activation, reduced-order models and nonlinear normal modes. Nonlinear Dyn. 48(3), 253-274 (2007) 
30. Srinil, N., Rega, G., Chucheepsakul, S.: Large amplitude three-dimensional free vibrations of inclined sagged elastic cables. Nonlinear Dyn. 33(2), 129-154 (2003)

31. Nielsen, S.R.K., Kirkegaard, P.H.: Super and combinatorial harmonic response of flexible elastic cables with small sag. J. Sound Vib. 251(1), 79-102 (2002)

32. Berlioz, A., Lamarque, C.-H.: A non-linear model for the dynamics of an inclined cable. J. Sound Vib. 279(3-5), 619-639 (2005)

33. Wang, L., Zhao, Y.: Large amplitude motion mechanism and non-planar vibration character of stay cables subject to the support motions. J. Sound Vib. 237(1-2), 121-133 (2009)

34. Ibrahim, R.A.: Nonlinear vibrations of suspended cables. Part III. Random excitation and interaction with fluid flow. Appl. Mech. Rev. 57(6), 515-549 (2004)

35. Matsumoto, M., Saitoh, T., Kitazawa, M., Shirato, H., Nishizaki, T.: Response characteristics of rain-wind induced vibration of stay-cables of cable-stayed bridges. J. Wind Eng. Ind. Aerodyn. 57, 323-333 (1995)

36. Wang, L., Xu, Y.L.: Wind-rain-induced vibration of cable: an analytical model (1). Int. J. Solids Struct. 40, 1265-1280 (2003)

37. Macdonald, J.H.G., Larose, G.L.: Two-degree-of-freedom inclined cable galloping. Part 1. General formulation and solution for perfectly tuned system. J. Wind Eng. Ind. Aerodyn. 96, 291-307 (2008)

38. Szabelski, K., Warminski, J.: Parametric self-excited nonlinear system vibrations analysis with inertial excitation. Int. J. Non-Linear Mech. 30(2), 179-189 (1995)
39. Eissa, M., Amer, Y.A.: Vibration control of a cantilever beam subject to both external and parametric excitation. Appl. Math. Comput. 152(3), 611-619 (2004)

40. Abdelhafez, H.M.: Resonance of a nonlinear forced system with two-frequency parametric and self-excitations. Math. Comput. Simul. 66(1), 69-83 (2004)

41. Szabelski, K., Warminski, J.: Vibration of a non-linear selfexcited system with two degrees of freedom under external and parametric excitation. Nonlinear Dyn. 14(1), 23-36 (1997)

42. Warminski, J.: Nonlinear normal modes of a self-excited system driven by parametric and external excitations. Nonlinear Dyn. 61(4), 677-689 (2010)

43. Abdel-Rohman, M.: Effect of unsteady wind flow on galloping of tall prismatic structures. Nonlinear Dyn. 26, 231252 (2001)

44. Nayfeh, A.H., Mook, D.T.: Nonlinear Oscillations. Wiley, New York (1979)

45. Verhulst, F.: Methods and Applications of Singular Perturbations: Boundary Layers and Multiple Timescale Dynamics. Springer, New York (2005)

46. Nayfeh, A.H., Chin, C.M.: Perturbation Methods with Mathematica. Dynamic Press Inc., Blacksburg (1999)

47. Khanin, R., Cartmell, M., Gilbert, A.: A computerized implementation of the multiple scales perturbation method using mathematica. Compos. Struct. 76, 565-575 (2000)

48. Doedel, E.J., Oldeman, B.E.: AUTO-07P: continuation and bifurcation software for ordinary differential equation (2009) 